\title{
ALFABETIZACIÓN DIGITAL DE LOS ESTUDIANTES EGRESADOS DEL VII CICLO DE EDUCACIÓN BÁSICA REGULAR EN CHIMBOTE, ANCASH, PERÚ
}

\author{
DIGITAL LITERACY OF VII SEMESTER EDUCATION STUDENTS \\ IN CHIMBOTE, ANCASH, PERU
}

Juana Luz Montero Pereda ${ }^{1}$

\begin{abstract}
RESUMEN
Hoy en día la sociedad de la información en el mundo educativo genera competencias nuevas y determinantes a partir del conocimiento y aplicación de las tecnologías de la información y comunicación en el proceso enseñanza-aprendizaje, abriendo posibilidades reales a docentes y estudiantes para insertarse en el contexto educativo actual y sobrevivir con calidad de vida en la sociedad del siglo XXI. Desde esta perspectiva, resultó muy importante aproximarse al conocimiento de los niveles de competencia alcanzados por los docentes de nuestro medio y relacionar esta condición con los logros en el proceso de alfabetización digital de los estudiantes del quinto grado de educación secundaria. Por ello, este trabajo de investigación se orientó a establecer la relación entre la formación y utilización de mediaciones de las tecnologías de la información y la comunicación en el desarrollo de estrategias de aprendizaje y la alfabetización digital de los estudiantes egresantes de quinto grado de educación secundaria de las instituciones educativas con aula de innovación del distrito de Chimbote, provincia del Santa, Ancash (Perú), en el 2011.
\end{abstract}

PALABRAS ClAVE: Tecnologías de la información y la comunicación, enseñanzaaprendizaje, competencia docente, alfabetización digital, contexto educativo actual.

* Recibido: 20 de marzo del 2013; aprobado: 23 de abril del 2013.

1 Abogada. Licenciada en Educación Secundaria. Magíster en Educación con Mención en Docencia, Currículo e Investigación. Docente en la ULADECH Católica. Email: janilu_57@hotmail.com 


\begin{abstract}
Nowadays, the information society in the world of education generates new needs in terms of new competences in the teaching-learning processes, due to the information and communication technologies. This opens real possibilities for teachers and students to insert the current educational context and live with better standard of life in the demanding XXI century. This study aimed to establish the relationship between the teachers's training and use of the information and communication technologies in classroom and the fifth year high school students's digital literacy in schools with innovation classrooms in Chimbote.
\end{abstract}

KEY WORDS: Information and communication technologies, teaching and learning, teaching competency, digital literacy, current educational context.

\title{
INTRODUCCIÓN
}

Los retos del siglo XXI conducen a una educación sensible a los cambios tecnológicos, a los sistemas de información y de acceso al conocimiento, a las formas de desarrollo científico y de innovación y a los nuevos significados de la cultura, para lograr un desarrollo económico equilibrado, que asegure la reducción de la pobreza, las desigualdades y la falta de cohesión social. En las sociedades modernas, las nuevas tecnologías de la información y la comunicación (TIC), adquieren un significado preponderante ya que la abundancia de información es casi infinita e imposible de asimilar, la complejidad del conocimiento es cada vez más grande, el tiempo de los individuos se convierte en recurso escaso y costoso determinando que los valores y actitudes de las nuevas generaciones cambien con las tendencias de la internacionalización, de los saberes y la globalización de la economía.

Es por ello que, desde una concepción pedagógica, utilizar las TIC en el proceso de formación profesional del educador implica, ante todo, definir un conjunto de presupuestos didácticos que garanticen una aplicación efectiva de la tecnología, en correspondencia con la intención de propiciar un aprendizaje innovador y desarrollador, fomentando una actitud independiente y creativa del estudiante. Supone, por tanto, las relaciones esenciales que integren un modelo pedagógico del uso de las TIC en la universalización.

\section{OBJETIVO GENERAL}

Establecer la relación entre la aplicación de mediaciones TIC en el desarrollo de estrategias de aprendizaje en los procesos de enseñanza-aprendizaje y los logros en la alfabetización digital de los egresantes de quinto grado de educación secundaria de las instituciones educativas que cuentan con aulas de innovación, en el distrito de Chimbote, Ancash (Perú). 


\section{OBJETIVOS ESPECÍFICOS}

1. Describir el nivel de aplicación de mediaciones TIC en las estrategias de aprendizaje, desarrolladas por los estudiantes de quinto grado de educación secundaria.

2. Describir los logros en alfabetización digital de los estudiantes de quinto grado de educación secundaria.

3. Estimar el nivel de competencia en TIC de los docentes del nivel secundario y su uso en el proceso de enseñanza-aprendizaje de las áreas curriculares.

4. Determinar la coherencia entre la aplicación de mediaciones TIC en el desarrollo de estrategias de aprendizaje y el logro en la alfabetización digital de los estudiantes de quinto grado de educación secundaria de las instituciones educativas, con aula de innovación, en el distrito de Chimbote.

\section{MATERIALES Y MÉTODOS}

La investigación estuvo planteada bajo el paradigma empírico-analítico, cuyo fundamento está en el positivismo lógico y su propósito es explicar, predecir, controlar los fenómenos, verificar teorías y leyes para regular los fenómenos estudiados.

Dentro del enfoque metodológico empírico-analítico, este estudio se propuso como una investigación del tipo descriptivo en el nivel ex post facto y correlacional. Dado que se buscó determinar los niveles de aplicación de mediaciones TIC en el desarrollo de estrategias de aprendizaje y su relación con los logros en los niveles de la alfabetización digital de los estudiantes que egresan de secundaria, en las instituciones educativas estatales con aula de innovación. Asimismo, y a manera de contextuar los aprendizajes de mediaciones TIC aplicados en la alfabetización digital, se estudió al docente en el campo de competencia TIC y su uso dentro de los procesos de enseñanza, en las áreas y talleres curriculares de secundaria. Es así que esta investigación se planteó descriptiva, dado que se trabajó sobre realidades de hecho; buscaba desarrollar una imagen fiel (descripción) del fenómeno planteado en el problema. En este caso, el énfasis estuvo en el estudio independiente de cada variable, característica que se sometió a investigación, desde la unidad de análisis del colectivo de estudiantes. Esto posibilitó que se integren las mediciones de las dos variables a nivel de correlación, con el fin de determinar 
cómo es o como se manifiesta el fenómeno de la aplicación de mediaciones TIC en las estrategias de aprendizaje y su relación con el nivel de alfabetización digital alcanzado por los egresantes de quinto grado de secundaria. Fue ex post facto, en tanto que el estudio no modificó, ni intervino directamente en el fenómeno sino que lo describió después de ocurrido.

La observación descriptiva del fenómeno sobre la aplicación de recursos de aprendizaje con mediación TIC en áreas curriculares y su relación con el nivel de alfabetización digital logrado por los estudiantes egresantes de quinto grado de secundaria de las instituciones educativas con aula de innovación se grafica de la siguiente manera:

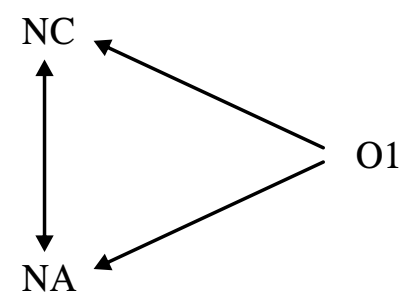

Donde:

O1: Observación de estudiantes en cada una de las variables.

NC: Utilización de recursos de aprendizaje con mediación TIC en áreas y talleres curriculares. Recursos TIC para reforzamiento bajo vigilancia del docente (aplicación de recursos Web 2.0 con autonomía funcional).

NA: Nivel de alfabetización básico y avanzado.

Son dos variables de estudio, en cuanto la unidad de análisis es el colectivo de estudiantes:

1. Utilización de recursos de aprendizaje con mediación TIC en áreas curriculares; y,

2. Nivel de alfabetización digital de los estudiantes egresantes del quinto grado de educación secundaria.

Una variable contextual como unidad de análisis, en cuanto corresponde al colectivo de docentes que interactúan con los estudiantes de quinto de secundaria en los procesos de enseñanza-aprendizaje de las áreas curriculares: Competencia en TIC y su aplicabilidad al proceso de enseñanza-aprendizaje. 
La población universo estuvo constituida por 1615 estudiantes y 121 docentes, de doce instituciones educativas que cuentan con aulas de innovación, dotadas por el Estado con TIC, en el distrito de Chimbote, en el año 2011. La muestra fue de 91 estudiantes y 60 docentes.

Se utilizó un muestreo estratificado no probabilístico y para el análisis interpretativo de la información se utilizaron, como referentes, los indicadores establecidos en la matriz de operacionalización de variables. Para el análisis de la información de la encuesta a los colectivos de docentes y estudiantes egresantes de quinto grado educación secundaria de las instituciones educativas con aula de innovación del distrito de Chimbote, se utilizó el programa estadístico SPSS versión 15.00 utilizando los baremos correspondientes.

\section{RESULTADOS Y DISCUSIÓN}

El 89\% de estudiantes de las instituciones educativas con aulas de innovación en Chimbote, aplicaron las TIC dentro de las estrategias de aprendizaje, bajo vigilancia del docente, y el $11 \%$ lo hicieron de manera autónoma.

Ello demostró una clara incidencia del docente en la centralidad en la enseñanza; paradigma tradicional que necesita urgentemente ser superado ya que interfiere y retrasa a los estudiantes en su inserción al mundo laboral y no cumple con los propósitos de la Educación Básica Regular al 2021, específicamente, el propósito $\mathrm{N}^{\circ} 11$ : Dominio de las Tecnologías de la Información y Comunicación (TIC), con el que se busca desarrollar en los estudiantes capacidades y actitudes que les permitan utilizar y aprovechar adecuadamente las TIC, dentro de un marco ético, potenciando el aprendizaje autónomo a lo largo de la vida. ${ }^{1}$

El $70 \%$ de estudiantes del quinto grado de educación secundaria de las instituciones educativas del distrito de Chimbote, con aula de innovación, solo alcanzaron un nivel de alfabetización digital mecánica y el 30\% avanzó hacia un nivel de alfabetización digital crítica. Esto implicó que las estrategias de enseñanza-aprendizaje utilizadas fueron producto de un trabajo basado en la racionalidad instrumental, práctico, rutinario y sin mayor esfuerzo mental, en desmedro del aprendizaje significativo, como lo concibe Vigotsky en su teoría del Constructivismo Social en la comprensión de los alumnos y su crecimiento cognitivo, "el hombre es un ser social por excelencia, que aprende por influencia del medio y de las personas que lo rodean; por lo tanto, el conocimiento mismo es un producto social y cultural; así, lo que asimila el individuo es fundamentalmente un reflejo de lo que pasa en la interacción social, en una sociedad determinada y una época histórica". ${ }^{2}$ 
La estimación del nivel de competencia en TIC de los docentes ha conducido los procesos de aprendizaje de los estudiantes sujetos de esta investigación a dos niveles. En cuanto al nivel de competencia del docente ante el dominio de las TIC, se encontró que el $46 \%$ no tuvieron competencia, $31 \%$ competencia básica y el $23 \%$ competencia avanzada; estimación muy importante, ya que evidenció el no reconocimiento, por parte de los docentes, que el "papel de los formadores no es tanto enseñar (explicar-examinar) unos conocimientos que tendrán una vigencia limitada y estarán siempre accesibles, como ayudar a los estudiantes 'a aprender a aprender' de manera autónoma, en la cultura de cambio y a promover su desarrollo cognitivo y personal mediante actividades críticas y aplicativas aprovechando la información y las potentes herramientas TIC". ${ }^{3}$

Con respecto a la frecuencia de utilización del aula de innovación del docente con sus estudiantes en el desarrollo del área curricular a su cargo, las respuestas mostraron poco uso de dicha mediación. Así, los resultados indicaron que el $11 \%$ asisten todas las semanas al aula de innovación con sus estudiantes y el $20 \%$, cada 15 días. Pero, si se agrupan los porcentajes "de vez en cuando" (23\%), y "no es necesario asistir" (26\%), demuestra que un $49 \%$ de docentes no reconocen que "Al asumir esta función, la responsabilidad del profesor va mucho más allá de la mera transmisión de conocimientos, (aunque todo lo que haga depende de su destreza para buscar nuevos conocimientos y para hacerlos accesibles al estudiante siempre que éste los necesite). Su interés se centra, sobre todo, en enseñar a descubrir esos nuevos conocimientos, a comprobarlos, asimilarlos y usarlos como base para otras experiencias de aprendizaje, para formar y modificar sus ideas y sus objetivos, y para tomar decisiones racionales. Más que una fuente o un proveedor, es alguien que guía hacia las fuentes, un organizador de las oportunidades de aprendizaje y un instructor en las técnicas de investigación y reflexión. Sus conocimientos no son un ingrediente en la educación del estudiante, quien debe aprehender y luego usar, sino más bien como un catalizador que promueve reacciones de aprendizaje y desarrollo como consecuencia del encuentro entre las capacidades humanas y el caudal creciente de conocimientos. ${ }^{4}$

Se comprobó que existía correlación significativa entre la aplicación de mediaciones TIC en el desarrollo de estrategias de aprendizaje y el nivel de alfabetización alcanzado por los estudiantes egresantes de quinto grado de educación secundaria, en instituciones educativas estatales con aula de innovación, del distrito de Chimbote, porque se observa una clara coherencia entre ambas dinámicas educativas. Pues, al no haberse desarrollado la alfabetización digital 
entre los estudiantes egresantes del nivel secundaria, tampoco se generó la transferencia de estos lenguajes hacia el crecimiento del potencial de aprendizaje que la tecnología imprime en este milenio, de la interacción y el empoderamiento participativo y autónomo a través de las redes informáticas que los estudiantes requieren como futuros ciudadanos.

\section{CONCLUSIÓN}

La transferencia de aprendizajes en torno a la Tecnología de la Información y la Comunicación entre docentes y estudiantes de quinto grado de educación secundaria, en las instituciones educativas con aula de innovación, en el distrito de Chimbote, muestra logros, pero también muchas y serias deficiencias, como el tener cuenta de facebook y el twitter o ambos o el correo electrónico y no compartir sus tareas, no navegar hipertextualmente, el no aplicar herramientas digitales y ofimática para recabar y utilizar la información, no interactuar ni colaborar con compañeros empleando entornos digitales para actividades educativas, igualmente no mostrar actitud positiva hacia el uso de la tecnología para el aprendizaje y la productividad en cuanto a tareas se refiere.

En el desarrollo de la multialfabetización en el ámbito de la formación del nivel secundario se observa poco dominio de la competencia en TIC de los docentes de las instituciones educativas con aula de innovación, lo que podría ser una limitación para el proceso formativo de sus respectivos estudiantes.

Las clases basadas en modelos didácticos tradicionales persisten y se trabajan día a día. En ellas se considera el uso de las TIC como un recurso más, no se valora la importancia que tienen para interpretar, ampliar y mejorar la calidad de lo aprendido.

El Ministerio de Educación no asume responsablemente la capacitación docente, peligrando el perfil en competencias que requiere del docente del siglo XXI.

\section{RECOMENDACIONES}

La sociedad de la información reta a las instituciones educativas a dotarse de recursos tecnológicos, tanto por el gobierno peruano como por otros organismos como Unesco, que refuerzan la urgencia de establecer desarrollos curriculares, en cuyos ejes transversales se definan, además de la lectura y de los valores cívicos, 
una trama formativa enraizada en el manejo y desarrollo aplicativo de las herramientas Web 2.0 y Web 3.0 mediadas por TIC.

El conocer y utilizar lenguajes y formas de comunicación para el desarrollo autónomo y la consiguiente aplicación al despliegue creativo de nuevas estrategias de aprendizaje, impulsa hacia una política de Estado y específicamente del gobierno local a ir más allá de proporcionar el hardware de esta tecnología, sino que valore fundamentalmente el software e intervenga en el proceso de formación continua del docente de todos los niveles educativos, a través de programas de actualización y reforzamiento en TIC, para el logro de la inserción de esta mediación en la cotidianeidad de los procesos de enseñanza y aprendizaje.

Convertirse en docentes modernos actuales e innovadores, porque los retos de este siglo exigen una educación con nueva concepción y práctica educativa direccionada a los cambios tecnológicos, a los sistemas de información, de acceso al conocimiento y a las formas de desarrollo científico, que finalmente son la razón de la cultura nueva que merecen nuestros estudiantes.

A los estudiantes, reconocer como su derecho una educación integrada con TIC, y como su deber, aprovechar al máximo su conocimiento, para lograr su inserción sin dificultades a la sociedad actual.

\section{REFERENCIAS}

1 Ministerio de EduCACión - Diseño Curricular Nacional de Educación Básica Regular - Propósitos de la Educación Básica Regular al 2021- Pág. 30 [En línea]: 15 de diciembre del 2008. [Fecha de consulta: 8 de abril 2013] Disponible en: [http://ebr.minedu.gob.pe/pdfs/ den2009final.pdf]

2 DIVISIÓN DE EDUCACIÓN SUPERIOR UNESCO - Las tecnologías de la información y comunicación en la formación docente- Guía de Planificación. Teorías que respaldan la nueva concepción acerca del proceso de aprendizaje- Pág. 30 [En línea]: 2004. [Fecha de consulta: 8 de abril del 2013] Disponible en: [http://www.unesco.org.uy/ci/fileadmin/comunicacióninformacion/lastecnologias.pdf]

3 MARQuÉs GRAELls, PERE. Los docentes: funciones, roles, competencias necesarias, formación. [En línea]: 7 de agosto del 2011 [Fecha de consulta: 8 de Abril del 2013] Disponible en: [http://peremarques.pangea.org/docentes.htm]

4 International CONFERENCE On EduCATION. XXXV Session. Ginebra, 1975: Final report Unesco. París. 1975 La cambiante función - del profesor. Perspectivas internacionales Cambio fundamental en la función docente: Enseñar a vivir en un momento de cambio. Pág. 57 [en línea]: 1975 [Fecha de consulta: 8 de Abril del 2013] Disponible en: [http://unesdoc.unesco.org/ images/0013/001360/136043so.pdf] 